\title{
The Role of Contrast-Enhanced Ultrasound in Evaluating Gestational Trophoblastic Neoplasia: A Preliminary Study
}

This article was published in the following Dove Press journal: Cancer Management and Research

\author{
$\mathrm{Na} \mathrm{Su} *$ \\ Chenyang Zhao $\mathbb{D D}^{*}$ \\ Bo Zhang \\ Zhenhong Qi \\ Bin Gao \\ Zhenzhen Liu (iD \\ Meng Yang \\ Yuxin Jiang \\ Qing Dai
}

Department of Ultrasound, Peking Union Medical College Hospital, Chinese Academy of Medical Sciences and Peking Union Medical College, Beijing, People's Republic of China

*These authors contributed equally to this work
Correspondence: Qing Dai; Yuxin Jiang Department of Ultrasound, Peking Union Medical College Hospital, Chinese Academy of Medical Sciences and Peking Union Medical College, Shuaifuyuan No.

I, Dongcheng District, Beijing 100730,

People's Republic of China

Tel +86 I0-69|5549|; +86 I0-69|55494

Email daiqing_pumch@163.com;

yuxinjiangxh@163.com
Objective: We aimed to compare the imaging features of gestational trophoblastic neoplasias (GTNs) and benign pregnancy-related uterine diseases on contrast-enhanced ultrasound (CEUS) to explore the clinical value of CEUS in differentiating these two types of uterine lesions.

Materials and Methods: In this prospective study, patients with GTNs before and after chemotherapy and benign pregnancy-related uterine lesions received conventional US and CEUS. The imaging features and parameters of CEUS of the lesions were assessed.

Results: A total of 73 patients were enrolled in this study, including 48 with GTNs and 25 with benign lesions. The enhancement pattern of newly diagnosed GTNs was mainly diffuse enhancement $(70.8 \%)$, with a non-enhanced area of less than $1 / 3$ of the entire lesion. For the benign group, the enhancement pattern was mainly ring-shaped enhancement (72.0\%), and the non-enhanced area was more than $1 / 2(68.0 \%)$, significantly different from that of GTNs $(P=0.000$ and 0.002 , respectively). Forty-one lesions demonstrated unclear boundaries on greyscale US but clearly displayed on CEUS. In both the benign and malignant groups, the measurements of lesion size on CEUS were larger than those on greyscale US $(P=0.000)$. The measurement differences $(\Delta \mathrm{D}: \mathrm{D} 2-\mathrm{D} 1)$ of the malignant cases were higher than those of the benign cases $(P=0.001)$.

Conclusion: GTNs and benign pregnancy-related uterine diseases have different imaging characteristics on CEUS. The boundary and involved range of the lesion can be clearly demonstrated on CEUS. CEUS possesses clinical value in diagnosing and evaluating GTNs and benign pregnancy-related diseases.

Keywords: ultrasound, contrast-enhanced ultrasound, gestational trophoblastic neoplasia

\section{Introduction}

Gestational trophoblastic neoplasia (GTN) is a category of malignant disease originating from abnormal trophoblastic proliferation and includes invasive hydatidiform mole, choriocarcinoma, placental site trophoblastic tumour (PSTT), and epithelioid trophoblastic tumour (ETT). Women of reproductive age are susceptible to GTN and are often referred to hospitals with symptoms of irregular vaginal bleeding after hydatidiform mole (HM), miscarriage, or childbirth and a continuous increase in $\beta$-human chorionic gonadotropin $(\beta-h C G)$. Invasive mole is the most common type of GTN, which usually follows with HM. Choriocarcinoma is a rather rare type of GTN, and it can occur after HM, abortion, or even normal pregnancies. PSTT and ETT are very rare malignant conditions, which can also 
occur after HM or normal gestations. ${ }^{1}$ GTNs are highly malignant, characterized by invasion into the uterine muscle layer and remote metastasis of abnormal trophoblastic cells at an early phase. If not treated in time and efficiently, GTNs can result in high mortality. Particularly, GTN poses a great threat to Asian women, who are reported to have a high rate of GTN according to previous studies., ${ }^{2,3}$ Importantly, GTN is very sensitive to chemotherapy, with a cure rate of $80-90 \%$. Therefore, early diagnosis and timely treatment are crucial for improving the prognosis of GTNs. ${ }^{4,5}$

The $\beta$-hCG level is the most important evidence for diagnosing GTN, and the monitoring of $\beta$-hCG level after HM can be crucial to identify postmolar GTN. Imaging methods provide additional information for diagnosing and evaluating GTN, and they also play a part in disease surveillance after treatment. ${ }^{6}$ Considering that GTNs are usually diagnosed clinically based on the integration of clinical, laboratory and imaging information, without reference to pathological results, in some conditions, misdiagnosis may occur. Ectopic pregnancies that occur in rare places, such as the uterine horn, caesarean section scar and intrauterine wall, as well as residual uterine pregnancies, can be misdiagnosed as GTNs and receive unnecessary chemotherapy. Another rare manifestation of GTNs is that they might present as uterine masses accompanied by abnormal vaginal bleeding after menopause and increasing $\beta$-hCG levels. Therefore, methods that can differentiate benign uterine lesions from GTNs are of clinical value.

Transvaginal ultrasound (US) has acquired a substantial role in diagnosing and managing GTNs with the development of Doppler techniques. ${ }^{7-9}$ GTNs and other benign pregnancy-related uterine diseases can sometimes appear similar on greyscale and colour Doppler US, manifesting as focal lesions with rich blood flow signals. ${ }^{10}$ Hence, it is crucial to develop new imaging methods as a complement to conventional US for diagnosing GTNs. Contrast-enhanced ultrasound (CEUS), a novel imaging technique that has been widely used clinically, can be used to visualize blood perfusion and microvessels of target organs and tissues with the use of microbubbles (MBs) as the contrast agent. By showing the distributions of blood vessels and real-time haemodynamic changes, CEUS can provide additional information for a variety of diseases, including some gynaecological diseases, ${ }^{11-13}$ thus enhancing the diagnostic efficacy of US. ${ }^{14,15}$ To our knowledge, the application of CEUS for GTNs has seldom been reported. In this study, CEUS was performed on patients with GTNs to identify the vascular distribution and blood perfusion of GTN lesions, aiming to investigate the feasibility of CEUS in diagnosing and evaluating GTNs. We also compared the imaging features of GTNs and benign pregnancy-related uterine diseases on CEUS to explore the clinical value of CEUS in differentiating the two types of uterine lesions.

\section{Materials and Methods}

This study was designed as a prospective study in a single medical center and approved by the Institutional Review Board of Peking Union Medical College Hospital (PUMCH). Written informed consent was received from all recruited patients. The study was conducted in accordance with the Declaration of Helsinki in the whole process.

\section{Patient Enrolment}

From September 2016 to July 2019, patients in the Department of Obstetrics and Gynecology of our hospital who met any of the following conditions and agreed to undergo a CEUS examination were recruited for this study: 1) patients who were diagnosed with GTN according to clinical standards (2002 The International Federation of Gynecology and Obstetrics [FIGO] standard for GTN) and whose uterine lesions were detected by conventional US; and 2) patients who were suspected of having pregnancy-related diseases and whose uterine lesions were detected by conventional US. Patients with severe heart disease and an allergy history were excluded. The final diagnosis of GTNs was verified by postoperative pathology or clinical standards if no operation was performed. The diagnosis of benign pregnancy-related diseases was verified by post-operative pathology. All the pathological examinations were verified by two experienced pathologists of our hospital.

\section{Settings for CEUS}

Greyscale US, colour Doppler US, and CEUS were performed using a transvaginal probe (V8-4, Philips IU22, USA) and a transabdominal probe (C5-2, Philips IU22). The scanning setting was maintained in the pre-set mode for each examination (Pelvic general for conventional US and Gyn/contrast general for CEUS). The settings for colour Doppler US included a pulse repetition frequency of $600-1000 \mathrm{~Hz}$, a wall filter of $45-55 \mathrm{~Hz}$, a maximum gain of $85-90 \%$, and a scale of $10 \mathrm{~cm} / \mathrm{s}$. The pulse 
inversion harmonics (PIH) technique was applied with a mechanical index of 0.08 .

The second-generation MB contrast agent SonoVue (Bracco, Italy) was used. Before injection, MBs were mixed with $5 \mathrm{~mL}$ of saline and shaken well. The mixture had a concentration of $8 \mu \mathrm{g} / \mathrm{mL}$ and a $\mathrm{pH}$ value of 4.5-7.5, isosmotic with human plasma.

\section{Imaging Procedures}

Transabdominal US was performed on patients with the following symptoms: 1) dynamic vaginal bleeding; and 2) large-sized lesions or lesions located at the bottom of the uterus that were difficult to visualize clearly by transvaginal US

Conventional greyscale US was first performed by a radiologist with 10 years of working experience in gynaecological US. The radiologist modified the settings, including gain, depth, and focus, to better visualize the lesions. Typical images of two perpendicular sections on greyscale US were recorded for each patient. Colour Doppler US was performed to observe the distributions and characteristics of blood flow within the myometrium and para-uterine tissues and vascularization of the surrounding and internal lesions.

Subsequently, a $21 \mathrm{G}$ trocar was punctured into the patient's left elbow vein to establish a venous channel, and then a bolus of $1.2 \mathrm{~mL} \mathrm{MB}$ suspension was rapidly injected into the venous channel with $5 \mathrm{~mL}$ saline. Each patient received 2-3 CEUS examinations. During the first examination, the lesions and the uterus were observed in overall view by moving the probe. The second examination was conducted after complete washout of the MBs. During the second examination, the sections showing areas with abundant vessels of the lesions were chosen for a consecutive observation for 3 minutes, the patients were instructed to breathe calmly, and the probe was kept fixed. CEUS was performed for a third time if no satisfactory video was obtained during the second examination. The whole CEUS process lasted for 20-30 minutes for each patient. The conventional US and CEUS examinations were performed by a radiologist with ten years of experience in gynaecological US.

\section{Imaging Analysis}

Two radiologists with five years of experience in gynaecological US read and evaluated the conventional US and CEUS images independently and were blinded to the clinical information. When discrepancies occurred, the final result was reached after a discussion between the two radiologists.

The following features were evaluated on conventional US:

1. site (uterine cavity, fundus, anterior wall, posterior wall, sidewall, uterine horn, and cervix);

2. size (maximum diameter of the lesion);

3. morphology (regular and irregular);

4. border (clear and unclear);

5. internal echo (no echo, hypoechogenicity, isoechogenicity, and hyperechogenicity);

6. composition (cystic, cystic solidity, and solidity);

7. posterior acoustic feature (no change, enhancement, and attenuation);

8. relationship with the serous membrane layer (clear boundary, unclear boundary, and breakthrough the serous membrane layer);

9. vascularization (no blood flow, sparse, and abundant); and

10. distribution site (peripheral, internal, and peripheral and internal).

The following features were assessed on CEUS:

1. enhancement order (concentricity, eccentricity, and simultaneous);

2. enhancement size (whether consistent with the size of greyscale US);

3. enhancement boundary (clear and unclear);

4. non-enhanced area (0, less than 1/3, 1/3-1/2, 1/2-2/ 3 , and more than $2 / 3$ ); and

5. enhancement pattern (diffuse enhancement, enhancement was observed within the whole lesion or with the fissured non-reinforced area; regional enhancement, some regions of the lesion were enhanced, with sheet-like unenhanced areas; reticulate enhancement, reticulate enhancement was observed, and the non-enhanced areas presented as a honeycomb structure; and ring-shaped enhancement, ring-shaped or semi-ring-shaped enhancement around the lesion was observed, with no internal enhancement or regional enhancement (Figure 1).

The time-intensity curve of CEUS was depicted using the off-line software QLAB (version 4.2.1, Philips). The lesion and the uninvolved myometrium symmetrical with the lesion were selected as the regions of 

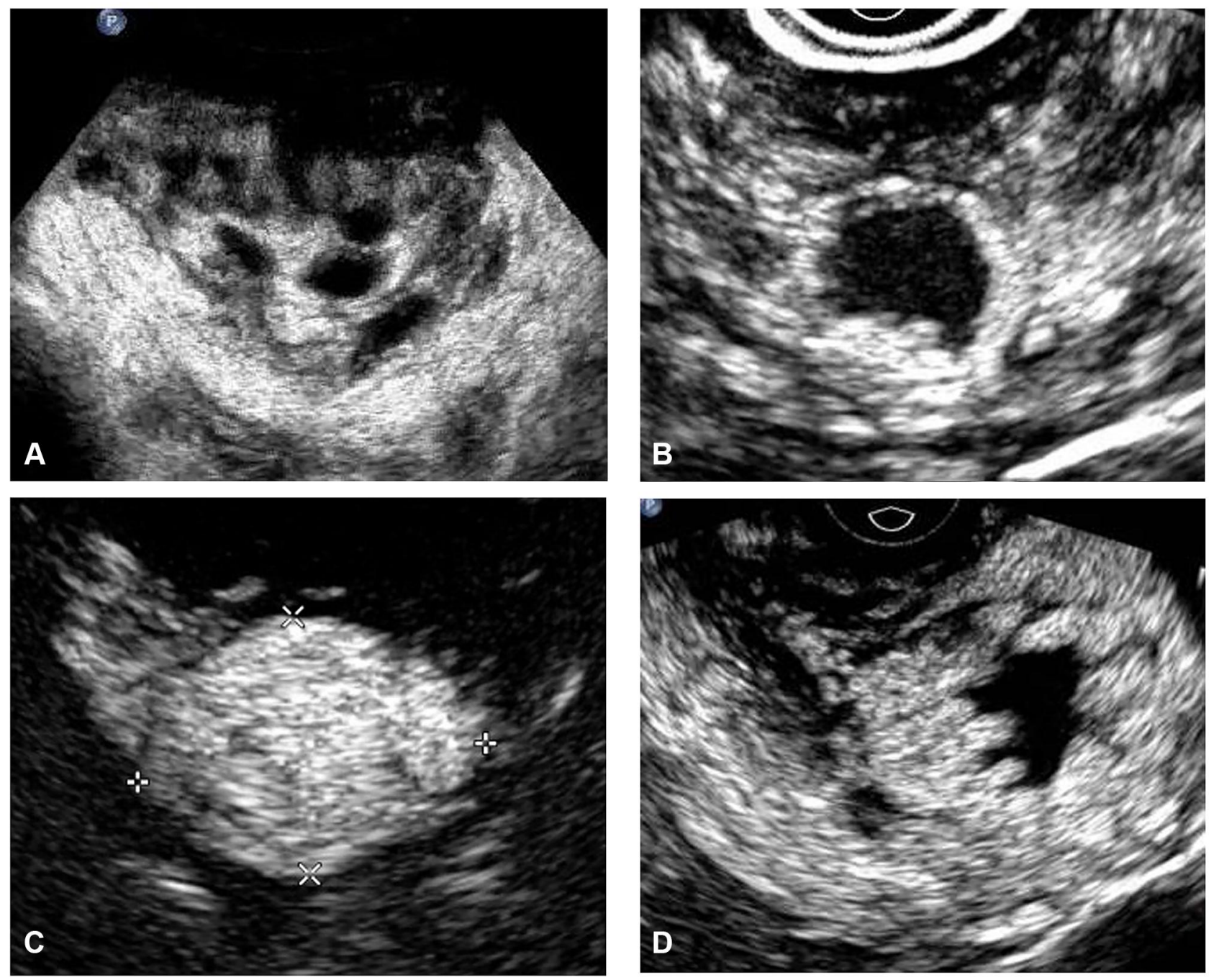

Figure I The four enhancement patterns (A) diffuse enhancement; (B) regional enhancement; (C) reticulate enhancement; (D) ring-shaped enhancement.

interest (ROIs). A rectangular frame of $2-3 \mathrm{~mm}^{2}$ was applied to sample the ROIs. When drawing the ROIs, large blood vessels were avoided. The imaging parameters of the lesions and uninvolved myometrium, including the start time of enhancement, peak time, rise time (peak time - start time), peak intensity, and enhancement rate (enhancement intensity/rise time), were calculated.

\section{Statistical Analysis}

The statistical analysis was performed using SPSS 20.0 (IBM Corporation, Armonk, NY, USA). Continuous variables are presented as the mean $\pm \mathrm{SD}$. The $t$-test and Mann-Whitney $U$-test were used to examine group differences in the mean values of imaging parameters. Continuous variables were compared using the $\chi^{2}$ test and Fisher's exact probability method. A $P$ value of $<0.05$ was considered statistically significant.

\section{Results}

A total of 73 patients were enrolled in this study. Forty-eight patients with GTNs received CEUS: 24 newly diagnosed patients who did not receive treatment and 24 patients who received 1-3 courses of chemotherapy before being referred to our hospital. There were 19 cases that received surgeries, including 17 that were treated with total hysterectomy, and 2 cases that received surgical excision. Seven cases were invasive hydatidiform mole, 11 of choriocarcinoma, and 1 of PSTT. The others had no pathological results and were diagnosed according to clinical standards. Four newly diagnosed patients with invasive 
hydatidiform mole and two newly diagnosed patients with choriocarcinoma received a CEUS examination again after the second course of chemotherapy. One newly diagnosed patient with invasive hydatidiform mole received two additional CEUS examinations after the second and fourth courses of chemotherapy. A total of 56 CEUS examinations, including 28 transabdominal US examinations and 28 transvaginal US examinations, were conducted for all patients with GTN. Twenty-five patients with benign pregnancyrelated uterine diseases underwent 8 transabdominal US examinations and 17 transvaginal US examinations. The clinical information of these patients is listed in Table 1. The patients were divided into three groups for further analysis: Group 1, patients with newly diagnosed GTNs; Group 2, patients with GTNs who received chemotherapy; and Group 3, patients with benign pregnancy-related uterine diseases.

Table I Patient Clinical Characteristics

\begin{tabular}{|c|c|c|}
\hline GTN & & \\
\hline Total number & & 48 \\
\hline Age & & $32.4 \pm 9.2$ \\
\hline Types of GTNs & Invasive hydatidiform mole & 26 \\
\hline & Newly diagnosed & 5 \\
\hline & After chemotherapy & 11 \\
\hline & Choriocarcinoma & 21 \\
\hline & Newly diagnosed & 8 \\
\hline & After chemotherapy & 13 \\
\hline & PSTT & 1 \\
\hline Stage (FIGO standard) & I & II (22.9\%) \\
\hline & II & $2(4.2 \%)$ \\
\hline & III & $33(68.8 \%)$ \\
\hline & IV & $2(4.2 \%)$ \\
\hline Prognosis score & Low risk $(\leq 6)$ & 28 \\
\hline & High risk $(>6)$ & 20 \\
\hline Benign pregnancy-relate & diseases & \\
\hline Total number & & 25 \\
\hline Age & & $30.4 \pm 4.6$ \\
\hline Types & Ectopic pregnancy & 18 \\
\hline & Cornual pregnancy & 11 \\
\hline & Scar pregnancy & 5 \\
\hline & Intramural pregnancy & 2 \\
\hline & Pregnancy residuals & 7 \\
\hline
\end{tabular}

Abbreviations: GTN, gestational trophoblastic neoplasia; FIGO, The International Federation of Gynecology and Obstetrics.

\section{Imaging Features on Conventional US}

The imaging features of Groups 1-3 are shown in Table 2. There was a significant difference in lesion boundary between Group 1 and Group $3(P=0.000)$; the malignancies were more likely to have unclear boundaries $(87.5 \%)$, while clear boundaries were observed in most patients with benign diseases (64\%). Malignant GTNs had more abundant vessels, of which blood vessels could be observed both within and surrounding the lesions (70.8\%). Most benign cases had a peripheral distribution of blood vessels. Compared with newly diagnosed GTNs, the patients that received chemotherapy exhibited fewer blood vessels, mostly surrounding the lesions. There were no differences in shape, echogenicity, or composition among the three groups.

\section{CEUS Features of Groups I-3}

The imaging features of the malignant GTNs and benign uterine diseases observed on CEUS are shown in Table 3, and the typical cases are illustrated in Figures 2-4.

Group 1: For the 15 cases of invasive hydatidiform mole, 93.3\% (14/15) demonstrated clear boundaries after CEUS, $80.0 \%(12 / 15)$ showed a diffuse enhancement pattern, $86.7 \%$ (13/15) showed simultaneous enhancement, and 80.0\% (12/ 15) had a non-enhanced area of less than $1 / 3$ of the whole lesion. For the 8 cases of choriocarcinoma, $75 \%(6 / 8)$ had clear boundaries. The diffuse enhancement pattern and ringshaped enhancement pattern accounted for 50\% (4/8) each, and $87.5 \%(7 / 8)$ of the lesions were enhanced simultaneously with varied portions of non-enhanced areas. Four cases showing a diffuse enhancement pattern had an average maximal diameter of $3.28 \pm 1.45 \mathrm{~cm}$ on CEUS images. The other 4 cases showing a ring-shaped enhancement pattern had an average maximal diameter of $5.80 \pm 1.48 \mathrm{~cm}$, which was significantly larger than those with a diffuse pattern. The case of PSTT showed a diffuse enhancement pattern and was simultaneously enhanced with a non-enhanced area of less than $1 / 3$ of the whole lesion.

Group 2: For the 17 cases of invasive hydatidiform mole after chemotherapy, 94.1\% (16/17) showed a clear boundary, $64.7 \%$ (11/17) showed a diffuse enhancement pattern, and $70.6 \%(12 / 17)$ were enhanced simultaneously with varied portions of non-enhanced areas. For the 15 cases of choriocarcinoma after chemotherapy, 93.3\% (14/ $15)$ showed clear boundaries, $73.3 \%$ (11/15) showed ringshaped enhancement, $73.3 \%$ (11/15) were enhanced simultaneously, and $86.7 \%(13 / 15)$ had non-enhanced areas greater than $2 / 3$ of the whole lesion. 
Table 2 Comparisons of Conventional US Features among Three Groups (Newly Diagnosed GTNs, GTNs After Chemotherapy, Benign Pregnancy-Related Uterine Diseases)

\begin{tabular}{|c|c|c|c|c|}
\hline \multicolumn{2}{|c|}{ Imaging Features of Conventional US } & \multirow{2}{*}{$\begin{array}{l}\text { Newly Diagnosed GTNs (\%) } \\
\mathbf{n}=24\end{array}$} & \multirow{2}{*}{$\begin{array}{l}\text { GTNs After Chemotherapy (\%) } \\
\mathrm{n}=32\end{array}$} & \multirow{2}{*}{$\begin{array}{l}\text { Benign Diseases (\%) } \\
\mathbf{n}=\mathbf{2 5}\end{array}$} \\
\hline & & & & \\
\hline Size $(\mathrm{cm})$ & & $4.1 \pm 2.2$ & $3.9 \pm 2.8$ & $4.0 \pm 1.8$ \\
\hline \multirow[t]{2}{*}{ Boundary } & Clear & $3(12.5)$ & $15(46.9)$ & $16(64.0)$ \\
\hline & Unclear & $21(87.5)$ & $17(53.1)$ & $9(36.0)$ \\
\hline \multirow[t]{2}{*}{ Shape } & Regular & $3(12.5)$ & $13(40.6)$ & $7(28.0)$ \\
\hline & Irregular & $21(87.5)$ & $19(59.4)$ & $18(72.0)$ \\
\hline \multirow[t]{2}{*}{ Internal Echogenicity } & Hypoechogenicity & $2(8.3)$ & $7(21.9)$ & $5(20.0)$ \\
\hline & Isoechogenicity & $22(91.7)$ & $25(78.1)$ & $20(80.0)$ \\
\hline \multirow[t]{2}{*}{ Composition } & Solid-cystic & $20(83.3)$ & $19(59.4)$ & $17(68.0)$ \\
\hline & Solid & $4(16.7)$ & $13(40.6)$ & $8(32.0)$ \\
\hline \multirow[t]{2}{*}{ Vascularization } & No/scarce & $0(0.0)$ & $10(31.2)$ & $3(12.0)$ \\
\hline & Abundant & $24(100)$ & $22(68.8)$ & $22(88.0)$ \\
\hline \multirow[t]{3}{*}{ Vascular distribution } & Internal & $3(12.5)$ & $19(59.4)$ & $17(68.0)$ \\
\hline & Peripheral & $4(16.7)$ & $2(6.2)$ & I (4.0) \\
\hline & Internal and peripheral & $17(70.8)$ & II (34.4) & $7(28.0)$ \\
\hline
\end{tabular}

Group 3: For the 11 cases of cornual pregnancy, all showed a ring-shaped enhancement pattern (100\%), and $72.7 \%(8 / 11)$ were enhanced simultaneously with varied portions of non-enhanced areas. For the 5 cases of scar pregnancy, all showed a ring-shaped enhancement pattern $(100 \%)$ and were enhanced simultaneously $(100 \%)$, and $80 \%(4 / 5)$ had a non-enhanced area greater than $2 / 3$ of the whole lesion. One of the intramural pregnancies had a ring-shaped enhancement pattern with a non-enhanced area greater than $2 / 3$, and the other showed a diffuse enhancement pattern. Most cases of pregnancy residuals had regional enhancement (85.7\%), and all were enhanced simultaneously $(100 \%)$, with varied portions of nonenhanced areas.

\section{Comparisons of CEUS Features Among the Three Groups}

The comparisons of CEUS features of the three groups are listed in Table 4. Most of the benign and malignant diseases showed clear boundaries on CEUS. Simultaneous enhancement was mostly observed in both benign and malignant lesions. There was a significant difference between Group 1 and Group 3 in the enhancement pattern and non-enhanced area $(P=0.000$ and 0.002 , respectively). The enhancement pattern of Group 1 was mainly diffuse enhancement $(70.8 \%)$, and the non-enhanced area was mostly less than $1 / 3$. In the benign group, the enhancement pattern was mainly ring-shaped enhancement $(72.0 \%)$, and the non-enhanced area was more than $1 /$ $2(68.0 \%)$.

Compared with Group 2, which received treatment, the enhancement pattern of Group 1 was mainly regional and ring shaped (78.1\%), and the non-enhanced area was larger than that of Group 1, with $2 / 3$ of the non-enhanced area in $50 \%$ of cases.

\section{Comparisons of CEUS Imaging \\ Parameters Among the Three Groups}

The parameters for the time-intensity curves are shown in Table 5. All three groups had significant differences in the imaging parameters of normal myometrium $(P<0.05)$. Early and fast enhancement was observed in all lesions, with a higher peak intensity than that in normal myometrium. No significant differences in the parameters were detected among the three groups.

Among the 81 lesions, 41 demonstrated unclear boundaries on greyscale US, but on CEUS, the boundaries of the lesions were clearly displayed and distinguished from normal myometrium.

In both the benign and malignant groups, the measurements of lesion size on CEUS were larger than those on greyscale US, with significant differences $(P=0.000)$. The 


\begin{tabular}{|c|c|c|c|c|c|}
\hline 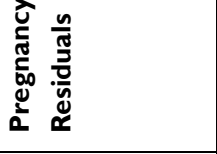 & II & 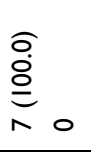 & 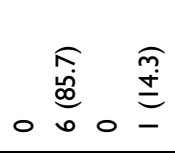 & o & 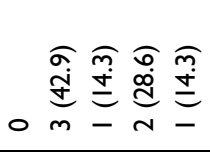 \\
\hline 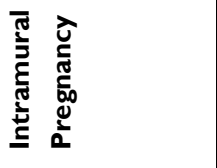 & II & 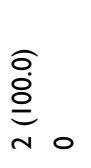 & 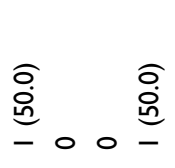 & 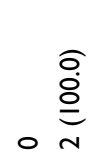 & 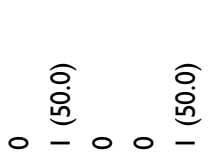 \\
\hline 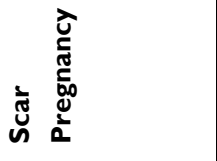 & ît & 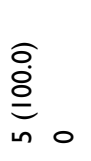 & $\begin{array}{llll} & & & \frac{0}{\stackrel{0}{\delta}} \\
0 & 0 & 0\end{array}$ & $\underset{0}{\stackrel{0}{\circ}}$ & 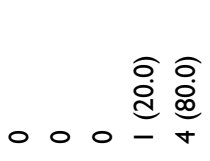 \\
\hline 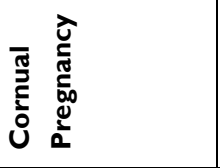 & $\overline{\bar{I}}$ & $\begin{array}{l}\hat{\sigma} \\
\dot{\alpha} \\
\stackrel{\bar{\sigma}}{0} \\
0\end{array}$ & 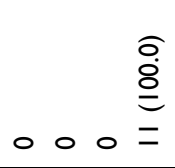 & 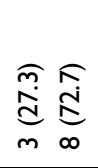 & 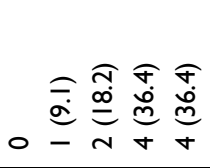 \\
\hline 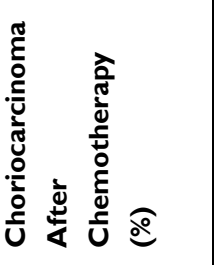 & $\stackrel{\llcorner}{\underline{n}}$ & 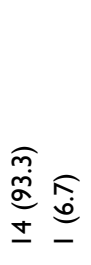 & 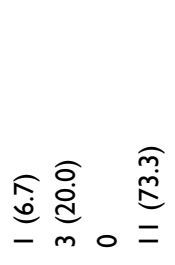 & 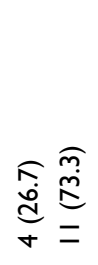 & 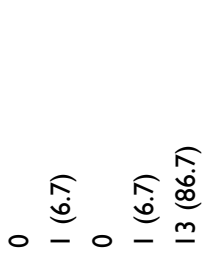 \\
\hline 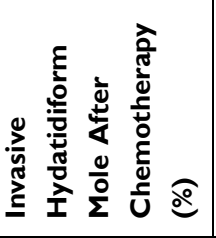 & $\bar{\pi}$ & 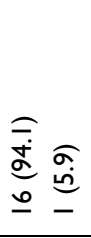 & 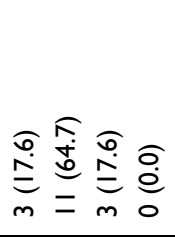 & 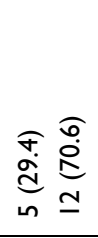 & 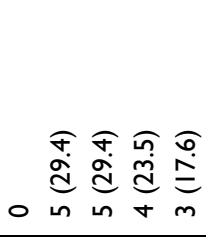 \\
\hline E & $\overline{\underline{I}}$ & 。 & $\begin{array}{l}\stackrel{o}{\dot{\theta}} \\
\stackrel{\text { on }}{=} 000\end{array}$ & o & 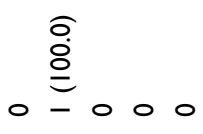 \\
\hline 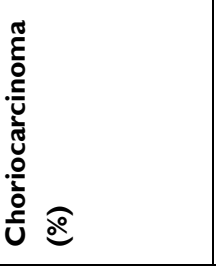 & $\stackrel{\infty}{11}$ & 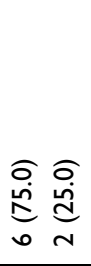 & 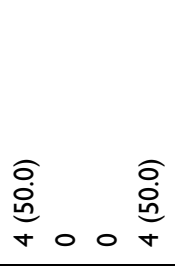 & 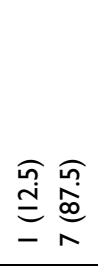 & 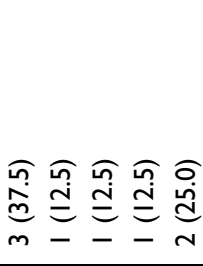 \\
\hline 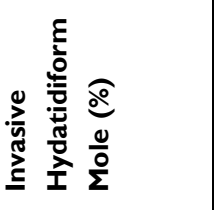 & $\frac{\underline{n}}{\underline{I}}$ & 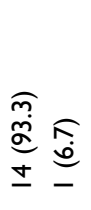 & 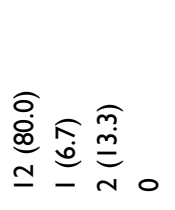 & 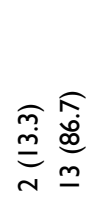 & 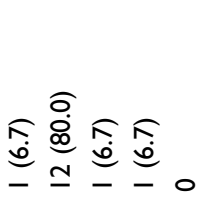 \\
\hline & & 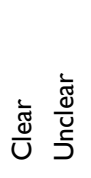 & 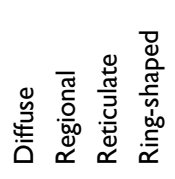 & 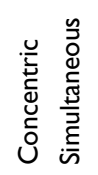 & 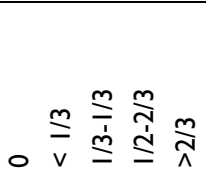 \\
\hline 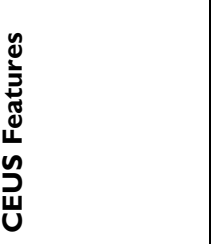 & & 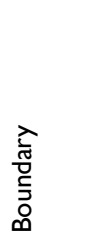 & 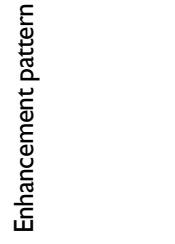 & 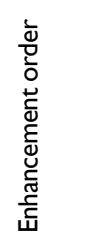 & 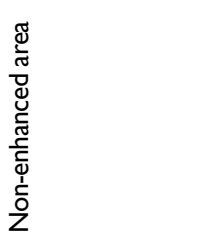 \\
\hline
\end{tabular}



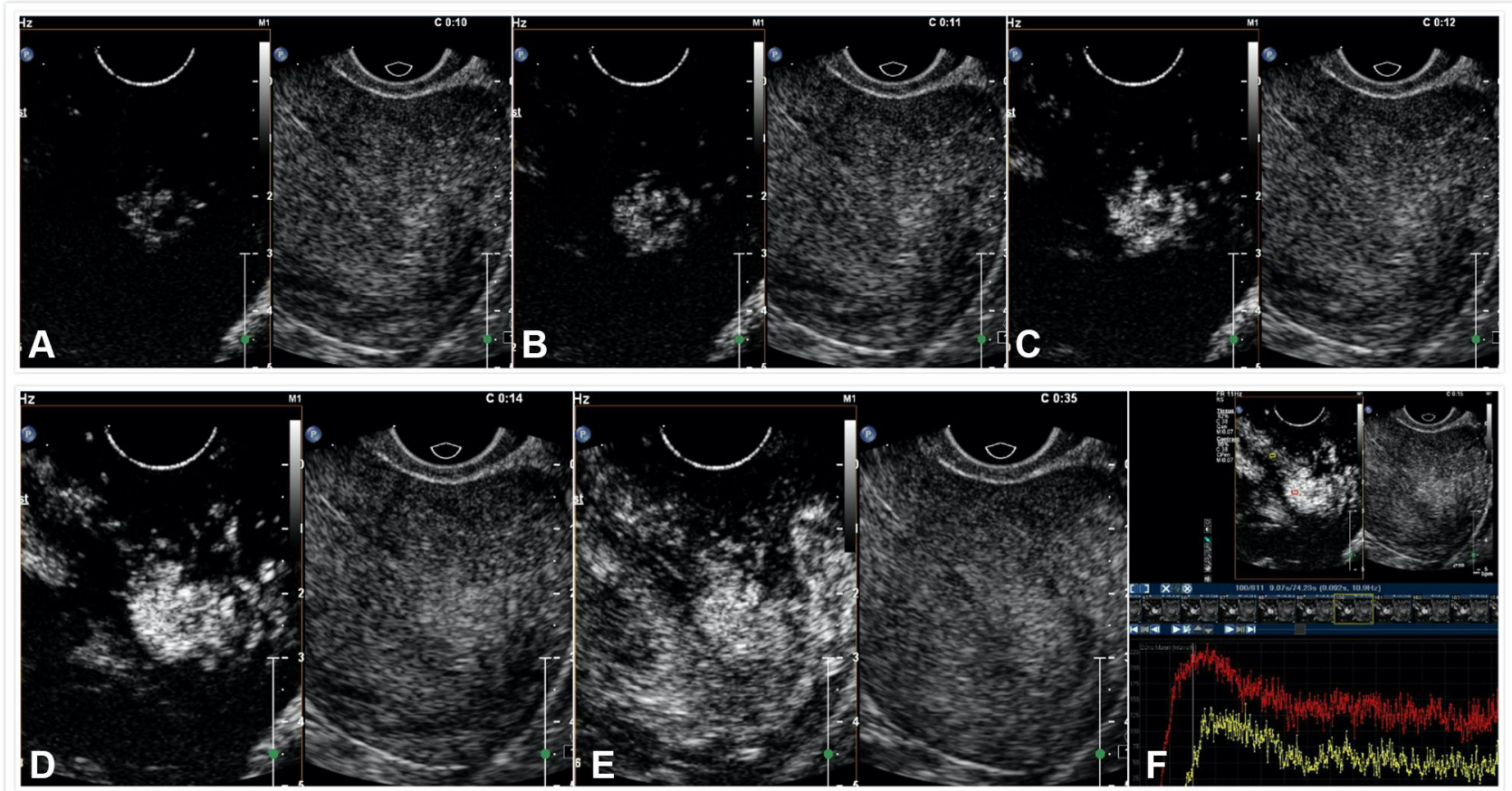

Figure 2 A typical case of choriocarcinoma. A 32-year-old female with abnormal vaginal bleeding for 52 days after delivery, was diagnosed with choriocarcinoma (Stage I) by clinical standard and post-operative pathology. (A-E) a greatly enhanced area within the posterior uterine wall was demonstrated on CEUS, with a diffuse enhancement pattern. (F) The time-intensity curve shows that the start time of the lesion is earlier than that of the normal myometrium, and the peak intensity is greater than that of the normal myometrium.
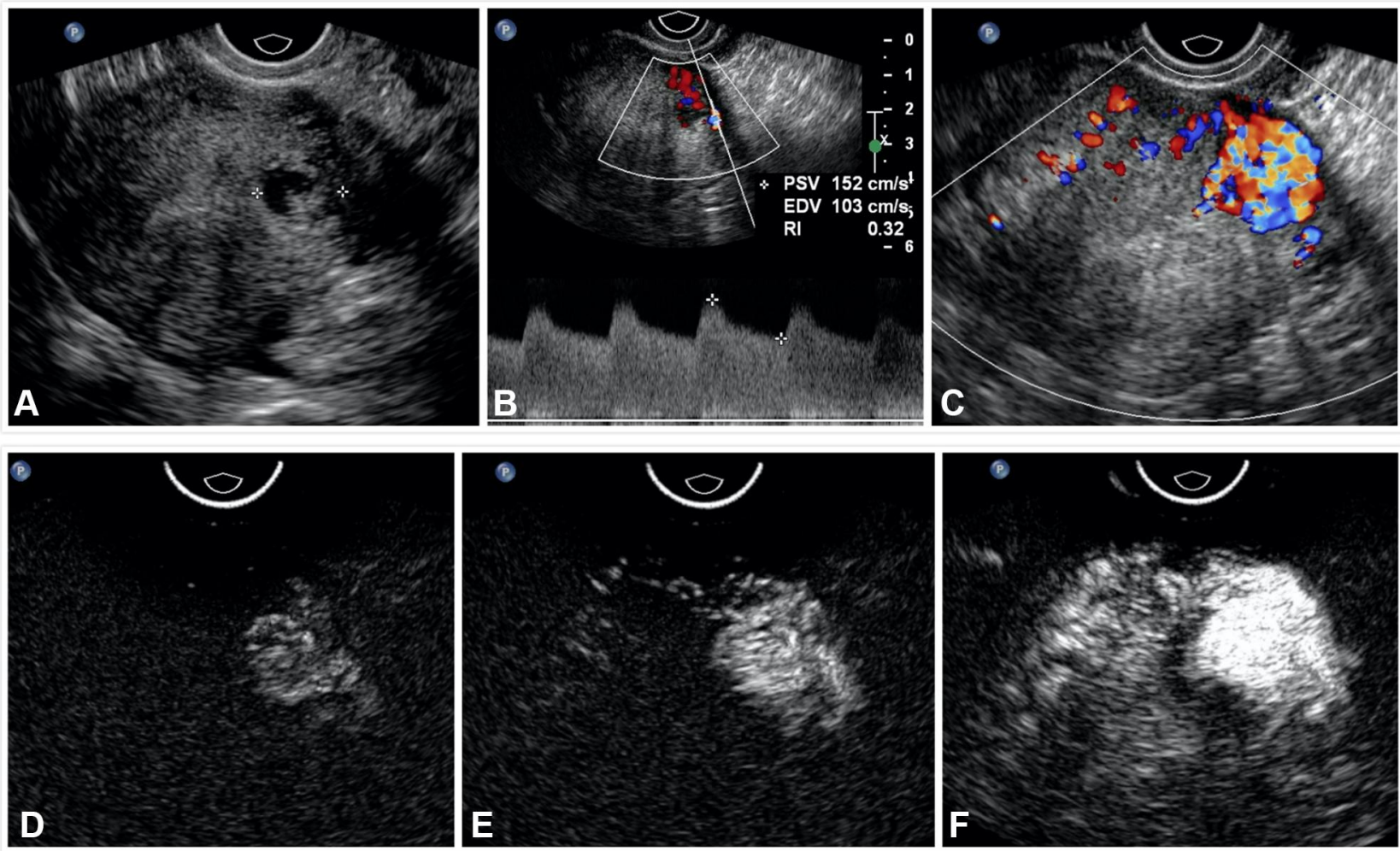

Figure 3 A typical case of invasive hydatidiform mole. A 28-year-old female with elevation of hCG level for 30 days after treatment of hydatidiform mole, was diagnosed as invasive hydatidiform mole according to clinical standard and post-operative pathology. (A) a cystic solid lesion was seen in the left wall of the uterus; (B and $\mathbf{C})$ abundant vessels were detected within the lesion by color-Doppler US, with a low resistance index (RI: 0.32); (D-F) a diffuse enhancement pattern of the lesion was observed on CEUS, earlier than the normal myometrium. The lesion boundary was more clearly visualized after enhancement, and the range of lesion displayed after CEUS was larger than grey-scale US. 

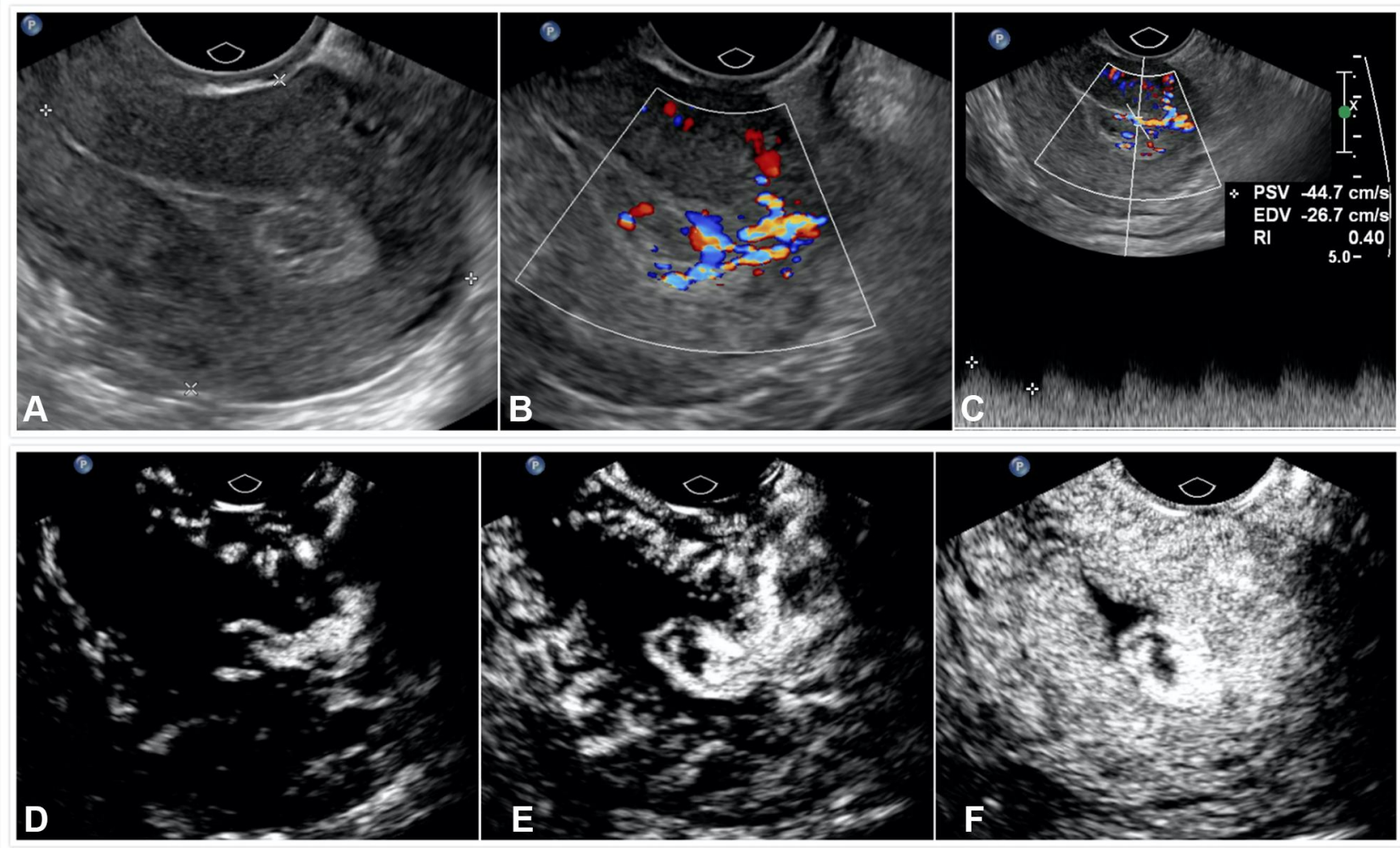

Figure $4 \mathrm{~A}$ typical case of benign pregnancy-related uterine lesion. A 35-year-old female with vaginal bleeding after abortion for more than I month, was diagnosed with pregnancy residue. (A) A heterogeneous mass was detected at the bottom of the uterine cavity; (B and $\mathbf{C}$ ) enriched blood flow signals with the mass were detected on color-Doppler US, with a low resistance index (RI: 0.40); (D-F) the lesion shows a regional enhancement pattern on CEUS with a non-enhanced area within the lesion, and the lesion boundary was more clearly visualized after enhancement.

measurement differences $(\Delta \mathrm{D}$ : D2-D1) of the malignant cases were higher than those of the benign cases $(P=$ 0.001) (Table 6).

\section{Discussion}

Using MBs as the contrast agent, CEUS can demonstrate microvessels and blood perfusion of the target organs or

Table 4 Comparisons of CEUS Features among Three Groups (Newly Diagnosed GTNs, GTNs After Chemotherapy, Benign Pregnancy-Related Uterine Diseases)

\begin{tabular}{|c|c|c|c|c|}
\hline \multicolumn{2}{|c|}{ Imaging Features of CEUS } & \multirow{2}{*}{$\begin{array}{l}\text { Newly Diagnosed GTNs (\%) } \\
n=24\end{array}$} & \multirow{2}{*}{$\begin{array}{l}\text { GTNs After Chemotherapy (\%) } \\
n=32\end{array}$} & \multirow{2}{*}{$\begin{array}{l}\text { Benign Diseases (\%) } \\
\mathrm{n}=\mathbf{2 5}\end{array}$} \\
\hline & & & & \\
\hline \multirow[t]{2}{*}{ Boundary } & Clear & $20(83.3)$ & $30(93.8)$ & $24(96.0)$ \\
\hline & Unclear & $4(16.7)$ & $2(6.2)$ & I $(4.0)$ \\
\hline \multirow[t]{4}{*}{ Enhancement pattern } & Diffuse & $17(70.8)$ & $4(12.5)$ & I (4.0) \\
\hline & Regional & $\mathrm{I}(4.2)$ & I4 (43.8) & $6(24.0)$ \\
\hline & Reticulate & $2(8.3)$ & $3(9.4)$ & $0(0.0)$ \\
\hline & Ring-shaped & $4(16.7)$ & II (34.3) & $18(72.0)$ \\
\hline \multirow[t]{2}{*}{ Enhancement order } & Concentric & $3(12.5)$ & $9(28.1)$ & $3(12.0)$ \\
\hline & Simultaneous & $21(87.5)$ & $23(71.9)$ & $22(88.0)$ \\
\hline \multirow[t]{5}{*}{ Non-enhanced area } & 0 & $4(16.7)$ & $0(0.0)$ & $0(0.0)$ \\
\hline & $<1 / 3$ & $14(58.4)$ & $6(18.8)$ & $5(20.0)$ \\
\hline & $1 / 3-1 / 3$ & $2(8.3)$ & $5(15.6)$ & $3(12.0)$ \\
\hline & $1 / 2-2 / 3$ & $2(8.3)$ & $5(15.6)$ & $7(28.0)$ \\
\hline & $>2 / 3$ & $2(8.3)$ & $16(50.0)$ & $10(40.0)$ \\
\hline
\end{tabular}


Table 5 CEUS Parameters of the Time-Intensity Curves for Three Groups

\begin{tabular}{|l|l|l|l|l|}
\hline \multicolumn{2}{|l|}{ CEUS Parameters } & Newly Diagnosed GTNs n=24 & GTNs After Chemotherapy n=32 & Benign Diseases n=25 \\
\hline Starting time (s) & Lesions & $9.83 \pm 2.76$ & $9.78 \pm 2.41$ & $10.50 \pm 2.48$ \\
& Myometrium & $12.75 \pm 3.18$ & $12.38 \pm 3.55$ & $13.82 \pm 2.76$ \\
\hline Peak time (s) & Lesions & $14.09 \pm 4.13$ & $14.37 \pm 2.77$ & $14.22 \pm 2.53$ \\
& Myometrium & $21.95 \pm 6.67$ & $18.59 \pm 5.10$ & $22.74 \pm 6.11$ \\
\hline Peak intensity (dB) & Lesions & $24.10 \pm 2.21$ & $23.01 \pm 2.99$ & $24.14 \pm 1.97$ \\
& Myometrium & $18.43 \pm 3.87$ & $17.91 \pm 4.47$ & $17.69 \pm 3.87$ \\
\hline Enhancement rate (dB/s) & Lesions & $6.96 \pm 3.35$ & $6.84 \pm 3.42$ & $6.93 \pm 1.79$ \\
& Myometrium & $2.65 \pm 1.52$ & $3.45 \pm 1.74$ & $2.38 \pm 0.99$ \\
\hline
\end{tabular}

Table 6 The Comparisons of Malignant and Benign PregnancyRelated Diseases in the Maximal Length Measured on Gray-Scale US and CEUS

\begin{tabular}{|l|l|l|l|}
\hline & $\begin{array}{l}\text { DI (Gray-Scale } \\
\text { US) }\end{array}$ & $\begin{array}{l}\text { D2 } \\
\text { (CEUS) }\end{array}$ & $\begin{array}{l}\Delta \text { D (D2- } \\
\text { D I) }\end{array}$ \\
\hline $\begin{array}{l}\text { Malignant }(n=56) \\
\text { Benign }(n=25)\end{array}$ & $4.47 \pm 2.58^{*}$ & $4.94 \pm 2.60^{*}$ & $0.47 \pm 0.29 * *$ \\
\hline
\end{tabular}

Notes: $* P=0.000 . * * P=0.001$.

tissues, thus providing additional imaging information for diagnosing diseases. Due to its non-invasive and non-toxic nature, CEUS has recently received wide utilization for a variety of clinical purposes. However, the use of CEUS in diagnosing GTNs and benign pregnancy-related diseases has seldom been reported. Makoto Emoto et al suggested that CEUS could be used to differentiate invasive and non-invasive GTNs by enhancing blood flow, ${ }^{16}$ but the detailed features of GTNs on CEUS have not yet been discussed. In this study, with reference to CEUS for liver, thyroid, and breast diseases, we investigated the characteristics of malignant and benign pregnancy-related uterine disease, including lesion boundary, enhancement order, enhancement size, enhancement pattern, and no enhancement area, on CEUS. This is the first study to compare the features of GTNs and benign pregnancy-related diseases on CEUS and validated the feasibility of CEUS in diagnosing and managing GTNs.

The blood supply of normal myometrium is characterized by radial distribution from the serosa to the intima. Therefore, it is speculated that the order of entry of MBs in GTNs should be from the outside to the inside. Simultaneous enhancement, which might be caused by the high velocity of blood flow in lesions that cannot be distinguished by visual observation, was observed in most lesions in this study. Lesions with concentric enhancement are generally large, and MBs take a long time to enter the entire lesion.

Most lesions, including those showing unclear boundaries on greyscale US, presented clear boundaries after the injection of MBs, which might account for the faster entry of MBs into lesions than into normal myometrium. Moreover, the lesion size measured on CEUS tended to be larger than that measured on greyscale US for both malignant and benign diseases. The measurement variances of malignant lesions were significantly larger than those of benign lesions. For the GTNs, an infiltrated area was formed between the muscular layer and the lesion without an envelope. These GTNs cannot be differentiated on greyscale US but can be distinguished by evident enhancement on CEUS; therefore, they were included in the measurement. For benign pregnancy-related diseases, there also exists an infiltrated area smaller than that of GTNs due to the minimal invasiveness of normal trophoblasts. The difference in enhancement patterns of GTNs and benign diseases can be accounted by the difference of degree of invasiveness of the two diseases. However, because both of the diseases are generated from the invasiveness of villous trophoblasts and the destruction of myometrium, to form blood pools and arteriovenous fistulas, the lesions would have high-speed and low-resistance changes on the spectral Doppler. Therefore, no significant difference was shown in CEUS parameters.

The newly diagnosed GTNs in this study exhibited mainly a diffuse enhancement pattern with no or minimal non-enhanced areas, reflecting abundant blood flow within malignant lesions. Ring-shaped enhancement and regional enhancement were observed mainly in benign lesions, representing the abnormal implantation site of trophoblasts. Relatively large areas of non-enhancement were detected in benign lesions. From the results of this study, 
we propose that the enhancement pattern may be used for differentiating malignant and benign pregnancy-related diseases. The four cases of invasive hydatidiform mole that presented reticulate enhancement were larger than other cases $(9.68 \pm 2.2 \mathrm{~cm}$ vs $3.87 \pm 1.39 \mathrm{~cm})$. For these four lesions, cystic structures were observed on greyscale US, representing oedema and villi congestion that occur in relatively large invasive hydatidiform mole lesions, and reticulate-enhanced regions represent the interstitial tissues of the villi. For the eight cases of choriocarcinoma, the average size of the lesions with diffuse enhancement was smaller than that of the lesions with ring-shaped enhancement $(3.28 \pm 1.45 \mathrm{~cm}$ vs $5.80 \pm 1.48 \mathrm{~cm})$, which could be explained by the occurrence of necrotic areas in large malignant lesions.

The malignant lesions that received chemotherapy also exhibited regional and ring-shaped enhancement with large non-enhanced areas, which also represents increasing necrotic areas within lesions after treatment. The CEUS manifestations after chemotherapy could also be useful axillary information to reflect the treatment efficacy and help guiding developing and changing treatment plans for the patients.

There are also several limitations to this study. First, the sample size of this study was relatively small. Some patients admitted to our hospital were transferred from other medical centres and received chemotherapy prior. More patients should be enrolled to further verify the role of CEUS in diagnosing GTNs and benign pregnancyrelated diseases. Although in this study, histological results were obtained for all the recruited cases, for cases nonmolar pregnancy, pathological examinations are not usually performed clinically. As a result, the number of cases recruited for this study is limited, and future verification of CEUS might also be hard in collecting cases. Secondly, in this study, the $\beta$-hCG levels of the patients were not discussed, for the focus of the study was mainly put on the imaging changes. In future research, imaging manifestations and parameters should be compared with the $\beta$-hCG levels, in order to better investigate the role of CEUS in the management of pregnancy-related diseases. Moreover, imaging methods have been attached with much importance by clinicians in the disease surveillance of GTNs. CEUS may also possess clinical potential in monitoring the treatment efficacy of different chemotherapy regimens and relapse of GTNs. Hence, further studies about the value of CEUS in disease monitoring of different regimens should be performed. More patients should be evaluated by CEUS before and after chemotherapy to investigate its role in reflecting the drug efficacy after comparing with clinical and laboratory results.

\section{Conclusion}

GTNs and benign pregnancy-related uterine diseases have different imaging characteristics on CEUS. CEUS can be utilized as an important adjunct tool for conventional US in diagnosing and evaluating GTNs before and after treatment by providing more diagnostic information about the vascularization and blood perfusion of lesions.

\section{Disclosure}

The authors declare no conflicts of interest.

\section{References}

1. Bruce S, Sorosky J. Gestational trophoblastic disease. In: StatPearls. Treasure Island (FL): StatPearls Publishing Copyright (C) 2020, StatPearls Publishing LLC; 2020. Available from: https://www.ncbi. nlm.nih.gov/books/NBK470267/. Accessed November 17, 2020.

2. Altieri A, Franceschi S, Ferlay J, et al. Epidemiology and aetiology of gestational trophoblastic diseases. Lancet Oncol. 2003;4:670-678. doi:10.1016/S1470-2045(03)01245-2

3. Steigrad SJ. Epidemiology of gestational trophoblastic diseases. Best Pract Res Clin Obstet Gynaecol. 2003;17:837-847. doi:10.1016/ S1521-6934(03)00049-X

4. Brown J, Naumann RW, Seckl MJ, et al. 15years of progress in gestational trophoblastic disease: scoring, standardization, and salvage. Gynecol Oncol. 2017;144:200-207. doi:10.1016/j.ygyno. 2016.08.330

5. Seckl MJ, Sebire NJ, Berkowitz RS. Gestational trophoblastic disease. Lancet. 2010;376:717-729. doi:10.1016/S0140-6736(10)60280-2

6. Shaaban AM, Rezvani M, Haroun RR, et al. Gestational trophoblastic disease: clinical and imaging features. Radiographics. 2017;37: 681-700. doi:10.1148/rg.2017160140

7. Cavoretto P, Cioffi R, Mangili G, et al. A pictorial ultrasound essay of gestational trophoblastic disease. J Ultrasound Med. 2020;39:5 97-613. doi:10.1002/jum.15119

8. Lin LH, Polizio R, Fushida K, et al. Imaging in gestational trophoblastic disease. Semin Ultrasound CT MR. 2019;40:332-349. doi:10.1053/j.sult.2019.03.002

9. Epstein E, Joneborg U. Sonographic characteristics of post-molar gestational trophoblastic neoplasia at diagnosis and during follow-up, and its relation to methotrexate resistance. Ultrasound Obstet Gynecol. 2020. doi:10.1002/uog.21971:

10. Zalel Y, Gamzu R, Lidor A, et al. Color Doppler imaging in the sonohysterographic diagnosis of residual trophoblastic tissue. $J$ Clin Ultrasound. 2002;30:222-225. doi:10.1002/jcu.10059

11. Liu D, Yang M, Wu Q. Application of ultrasonography in the diagnosis and treatment of cesarean scar pregnancy. Clin Chim Acta. 2018;486:291-297. doi:10.1016/j.cca.2018.08.012

12. Liu SQ, Xie X, Liu YP, et al. Uterine arteriovenous malformation combined with iliac arteriovenous malformation diagnosed by contrast-enhanced ultrasound: a case report and review of the literature. Clin Hemorheol Microcirc. 2019;73:293-298. doi:10.32 33/CH-180489

13. Lieng M, Qvigstad E, Dahl GF, et al. Flow differences between endometrial polyps and cancer: a prospective study using intravenous contrast-enhanced transvaginal color flow Doppler and three-dimensional power Doppler ultrasound. Ultrasound Obstet Gynecol. 2008;32:935-940. doi:10.1002/uog.6267 
14. Erlichman DB, Weiss A, Koenigsberg M, et al. Contrast enhanced ultrasound: a review of radiology applications. Clin Imaging. 2020;60:209-215. doi:10.1016/j.clinimag.2019.12.013

15. Wilson SR, Burns PN, Kono Y. Contrast-enhanced ultrasound of focal liver masses: a success story. Ultrasound Med Biol. 2020;46:1059-1070. doi:10.1016/j.ultrasmedbio.2019.12.021
16. Emoto M, Sadamori R, Hachisuga T, et al. Clinical usefulness of contrast-enhanced color Doppler ultrasonography in invasive and noninvasive gestational trophoblastic diseases: a preliminary study. J Reprod Med. 2011;56:224-234.

\section{Publish your work in this journal}

Cancer Management and Research is an international, peer-reviewed open access journal focusing on cancer research and the optimal use of preventative and integrated treatment interventions to achieve improved outcomes, enhanced survival and quality of life for the cancer patient.
The manuscript management system is completely online and includes a very quick and fair peer-review system, which is all easy to use. Visit http://www.dovepress.com/testimonials.php to read real quotes from published authors. 